\title{
The Emergence of Autonomous Representations in Artificial Agents
}

\author{
Argyris Arnellos \\ University of the Aegean/Department of Product and Systems Design Engineering, Hermoupolis, Greece \\ Email: arar@aegean.gr \\ Spyros Vosinakis, Thomas Spyrou and John Darzentas \\ University of the Aegean/Department of Product and Systems Design Engineering, Hermoupolis, Greece \\ Email: \{spyrosv, tsp, idarz \}@aegean.gr
}

\begin{abstract}
Representational autonomy is a key property of an artificial agent. The type of representational structures and the role they play in the preservation of an agent's autonomy are pointed out. The limitations of the traditional cognitivist approach and of the embodied intelligent approach to support such representational structures are described and indicated. A framework of self-organising Peircean semiotic processes is introduced and it is then applied to demonstrate the emergence of autonomous representations in an artificial agent interacting with the environment.
\end{abstract}

Index Terms-representation, autonomy, self-organisation, Peircean semiotics, emergence, agent.

\section{INTRODUCTION}

Contemporary research in artificial environments has marked the need for autonomy in artificial agents. Autonomy has many interpretations in terms of the field it is being used and analysed, but the majority of the researchers in artificial environments are arguing in favour of a strong and life-like notion of autonomy, which should first of all replace omniscience in artificial worlds. If believability is considered as a crucial factor, artificial agents should appear to be able to interact with other agents and users in unexpected events and circumstances under fallible anticipations (under incorrect information), to have limited perception capabilities and plausible action, to create and communicate new meanings about their environments and to exhibit novel interactions. Such agents could be used in dynamic and open-ended scenarios, where adaptability is needed, or even to be reused in a different environment. In [1] it is argued that the ideal case for an artificial agent would be to exhibit a human-like personality, and they considered this to be the combination of a completely realistic interaction with the environment and human-like cognitive abilities.

In an ALife perspective, an agent's personality should emerge from its open-ended interaction with the environment. To do so, there should be the prerequisites and the conditions for the agent to be structurally coupled to the environment, and to develop the respective lowand high-level cognitive capabilities through the whole history of its adaptation. This is the problem with agency in any context and autonomy seems to be one of its most essential properties.

In the next sections the type of representational structures and the role they play in the preservation of an agent's representational autonomy are pointed out. The limitations of the traditional cognitivist approach and the embodied intelligence approach to support such representational structures is described. A framework of self-organising Peircean semiotic processes is introduced and it is then applied to demonstrate the emergence of autonomous representational structures in an artificial agent interacting with the environment. This is an attempt to design artificial agents with enhanced representational autonomy, since this will be a fundamental advantage towards the design of strongly autonomous artificial agents.

\section{AutONOMY AND REPRESENTATIONS IN STRONG AGENCY}

Autonomy has many definitions in respect to the domain it is being used. The same is true regarding the notion of agency. In this paper, agency and autonomy are defined in their stronger notions, such as those usually applied to human agents. Hence, it can be said that an agent is a system which exhibits the fundamental properties of interactivity, which is considered as the ability to perceive and act upon its environment by taking the initiative; intentionality, which is considered as the ability to effect goal-oriented interaction by attributing purposes, beliefs and desires to its actions; and autonomy, which is the ability to operate intentionally and interactively based only on its own resources.

There is an interesting interdependence between the three fundamental properties. As it is suggested in [2], there is no function without autonomy, no intentionality without function and no meaning without intentionality. The circle closes by considering meaning (representational content) as a prerequisite for the maintenance of system's autonomy during its interaction. 
Following contemporary research coming from the realm of living systems and dynamic anticipatory systems, autonomy implies also a representational autonomy, which will provide the content based on which the system will be able to interact with the environment, in such a way, that will be able to maintain its own functional autonomy [2] [3]. This is something that seems to be widely observed in living systems and which implies their capacity for open-ended adaptation. Hence, the degree of autonomy depends on the type of representations and their content that drives an agent's functionality through its interactions with the environment. Moreover, the notion of representation is central to almost all theories of cognition, therefore being directly and indirectly connected with fundamental problems in the design of artificial cognitive agents [4]. Thus, in the following paragraphs we briefly describe the way the notion of representation is used in the wellknown frameworks of cognition, and the resulting limitations that are imposed in the autonomy of the virtual agents designed based on these frameworks.

The majority of artificial agents inhabiting into artificial environments are designed using architectures that are based on the cognitivist assumption [5], [6]. However, there are a lot of practical problems. Although these approaches have been successful on modelling very specific and well-defined tasks requiring high-level cognition, they have been quite unsuccessful on everyday tasks that humans find extremely easy to manage. In particular, cognitivist artificial agents fall into the trap of the symbol grounding problem and of the frame problem. Due to the disembodied nature of cognition the syntactic and semantic aspects of a cognitivist artificial agent are separated, thus making the creation and enhancement of inherent meaning structures almost impossible. Hence the symbol grounding problem [7]. The frame problem comes as a consequence. Specifically, since agent's functionality is based on predetermined representations, it will neither have the capacity to generalise its meanings in order to act on new contexts presenting similar relations and conditions, nor the ability to develop new representations and hence to function adaptively whenever is needed. There have been some attempts to ground representations in the sensorimotor interaction with the environment, but a cognitivist grounding theory should also explain the interdependence of the transducing system with its environment and the central computational system, in order to be complete [8]. Everything that an artificial agent does, if it is to be a system that exhibits autonomy, should be primarily intrinsically meaningful to itself. Cognitivist artificial agents do not have this ability.

A radical alternative to cognitivism and disembodiment in general is emergent and embodied cognition, where one is looking for autonomy and intelligence without representations [9]. This approach introduces numerous advantages, compared to the cognitivist assumption, for the shake of disregarding the need for system's representations. Symbolic representation disappears completely - the productive power of the agent's functionality is embodied within the network structure, as a result of its particular history. In this absence there is no need for symbol grounding, since the agent is now physically grounded to the environment. Additionally, embodiment seems as a way out of the frame problem, since the choice of the relevant model for the agent's functional organisation is now being constrained bottom-up by the environment [10].

The embodied approach comes with serious drawbacks. In agents based on the subsumption architecture [11], as complexity of the environment increases, there is a difficulty in the agent's sensorimotor organisation to handle the internal communication needed to coordinate its activity. Also, the ability for classification for merely dynamic and self-organising agents cannot transcend their attractor's landscape complexity, which in a way, restricts their meaning evolving threshold. Although an embodied agent seems to be able to handle very simple tasks with only primitive stimulus-response actions, its cognitive capabilities cannot scale to tackle more complex phenomena. This happens because physical grounding cannot generate internal content, which is deemed more than necessary when the environment, used as a model in itself, is just not enough. These are evidences that the use of representations, even in reflexive behaviors, becomes essential [12]. However, representations should not be generic, context-free and predetermined, but they should be an emergent product of the interaction between an agent and its environment [4].

\section{EMERGENT REPRESENTATIONS VIA SELF- ORGANISING SEMIOTIC PROCESSES}

There are many hybrid architectures which are trying to build autonomous agents, especially in virtual environments [13], [14]. In this works, autonomy is used in the very light sense of the word, and furthermore, there are some major problems, such as lack of scaling, the need of an external planner, etc. These attempts consider intelligence as a separate module which should be adapted to the other modules of the agent. In a strongly autonomous agent any representational functional organisation should be emergent on its interaction with the environment. Embodied artificial agents seem like a good candidate for meaning-based interaction, but their capabilities are limited. The complexity of an emergent self-organising system can only be enriched through its interaction (structural coupling) with other systems in its environment [15]. The immediate problem to overcome is to find the means by which this structural coupling will take place. Self-organising and embodied systems admit no functional usefulness to representations and they regard information only as socially ascribed to a process from other observers. On the other hand, it seems that all necessary information for an adaptive self-organising system must be embedded in its functional patterns, which are cross-correlated with incoming ones, in order to be built up or to dynamically form new ones in dependence with the system's anticipation [16]. Then, the incorporation of a process to support the vehicle of the 
representation which carries internal information about an external state seems imperative. This process should give the interactive dimension to the self-organising system and furthermore, it should correspond to the embedded structure of emergent representations. In this way, the cognitive system will preserve its self-organising status and the respective properties that come with embodiment, but it will also acquire a fruitful functionality for autonomy and open-ended adaptive interaction. Peircean semiosis can be seen as the process which drives the system into meaningful interaction. In [17] Peircean semiotic processes and especially Peircian triadic semiosis [18] are extensively presented as a proper framework in order to complement the interaction of selforganising systems in a dynamic information environment, as well as, the ability of such processes to model intentional interactions [19].

In the proposed framework, intelligence is not consider as an extra module, but as an asset emerging from the agent's functionality for interaction. Specifically, the use of the proposed framework aims at the unification of the modality of interaction, perception and action with the smallest possible number of representational primitives. The present attempt is in correspondence with contemporary works in AI, such as [20], where only the concept of semiotic symbol is used to ground lexicons in robots and [21] where semiotics and schema theory is used to ground language in action and perception of a robot. In the present paper, there is an attempt to design a more generic architecture which will integrate aspects of self-organisation and embodiment with Peircean semiotics. There is in no way a demonstration of a totally autonomous system, but the introduced architecture overcomes the symbol-grounding problem, which is the fundamental obstacle for the frame problem, and by doing so, it introduces a type of representational structures that are integrated into the functional structure of the artificial agent. This types of representations are more consistent with the demand for representational autonomy coming from dynamic anticipatory systems research [4]. Finally, the whole endeavour is in parallel with ideas relating research in cognitive science to AI systems, as these are outlined in [22].

\section{A. The Structure of Peircean Signs}

In Peirce's semiotic theory signs have a triadic structure constituted by three distinct interrelations (semiotic components). The triadic sign relation is never present in itself as a closed entity, but it is integrated into a sign process. Also, in order a sign to come into existence through a sign process there must exist a cognitive agent to support its interpretation. The meaning of a sign is not static but it is formed by a sequence of interpretants and it is virtually unlimited. Peirce also distinguishes between the Immediate Object (IO - the object as represented in the sign) and the Dynamic Object (DO - the object that the sign cannot express). In other words, a representamen is the sign-vehicle which can be implemented in any kind of structure and it is the form with which the signified object will be conveyed to the cognitive system. An object is the object to which the sign refers through the immediate object (IO) of the sign. The interpretant is the sense that the cognitive system made of the sign, which is a more developed sign.

Now, each one of the three sign relations (S-OR-IR) constituting the triadic structure may exhibit three different modes. The relation of the sign to itself ( $\mathrm{S}$ - the intrinsic relation of the sign) is constrained by the qualities that have been mediated by the DO to the signvehicle. There are three cases: a Qualisign (Q), when the representamen shares certain qualities with what it mediates to the cognitive system, a Sinsign (S), when the representamen realizes (at this very time) the form of what it mediates and a Legisign $(L)$ when the mediator's general pattern realizes the form of what it mediates.

The relation of the representation to the object (OR) is constrained by the cognitive agent. The latter determines, on the basis of her anticipations (habits and beliefs) and the characteristics of the sign, a kind of relation between the sign and a certain DO. Such a relation can be Iconic (Ic), where the sign has no dynamic connection with the DO but its qualities resemble those of the DO; Indexical (Id), where the sign has a causal relation to its DO; Symbolic (Sy), where the sign is related to the DO based on the agent's habits and beliefs (anticipations). The relation between the sign and its interpretant (IR) depends on the type of schematization the representamen exhibits in order to include these aspects of the sign which concern its relation to the interpretant. Here, depending on the type of schematization, one can find a Rheme (R), which is the most abstract relation between a sign and its interpretant, as it can only be assumed to represent a possible existence; a Dicent $(D)$, which is a proposition (sign of actual existence), it must necessarily contain a Rheme and it cannot be Iconic; an Argument (A), where the anticipations of the agent are the basis on which she knows that a certain sign can be connected with an interpretant [21].

The basic structural element of the proposed framework is the semiotic component. A possible representation is to use a frame-like structure, and to let individual slots express the respective qualities (qualisigns) of the object they represent. For indexing and interpretation purposes, two more slots should be reserved to describe the unique id of the component and the type of data it holds. In the case of virtual environments, possible objects that can be represented in the agent's knowledge base using semiotic components are:

- entities: the individual visual elements that exist as 3d geometries in the environment. The semiotic component should possibly contain their spatial properties (e.g. translation, rotation, bounding box size) and other custom qualities that better describe their nature.

- relations: spatial (e.g. near), structural (e.g. part-of) or other relations between entities. Spatial relations are usually iconic representations.

- situations: a collection of objects and relations between them that describes (part of) the environment 
- actions: Preconditions (described as the initial situation), performance (series of motor commands) and effects (changes between initial and final situation).

The slots can contain either crisp values or sets. In the latter case, the component describes not just one object but a category (legisign). A possible semiotic component representing an observed entity could be in the form presented in table 1.

TABLE I. AN EXAMPLE OF A SEMIOTIC COMPONENT

\begin{tabular}{|c|c|c|}
\hline s-id & 114 & $\begin{array}{c}\text { semiotic } \\
\text { component id }\end{array}$ \\
\hline s-type & 'entity' & $\begin{array}{c}\text { semiotic } \\
\text { component type }\end{array}$ \\
\hline id & 1 & entity id \\
\hline pos & $(1.0,0.5,3.4)$ & position vector \\
\hline rot & $(0.747,0.0,1.0,0.0)$ & orientation \\
\hline size & $(2.5,1.0,2.0)$ & bounding box size \\
\hline type & 'chair' & entity type \\
\hline legs & 4 & number of legs \\
\hline
\end{tabular}

\section{B. Self-organising Peircean Semiotic Processes}

The abstract architecture rising from the interaction of a self-organising system with its environment based on Peircean semiotic processes is shown in Fig. 1. A detailed analysis of the architecture is given in [23].

As a first step towards a computational methodology for implementing the proposed framework, an example has been set up, where agents are wandering around an environment and try to learn simple actions. The environment contains a number of agents and passive objects and has an embedded collision detection and response algorithm Each agent has its own abilities concerning perception and action and initially it has no representational structures regarding possible actions. The objective is to show that different grounded representations will emerge through the random interactions of the agents with the environment.

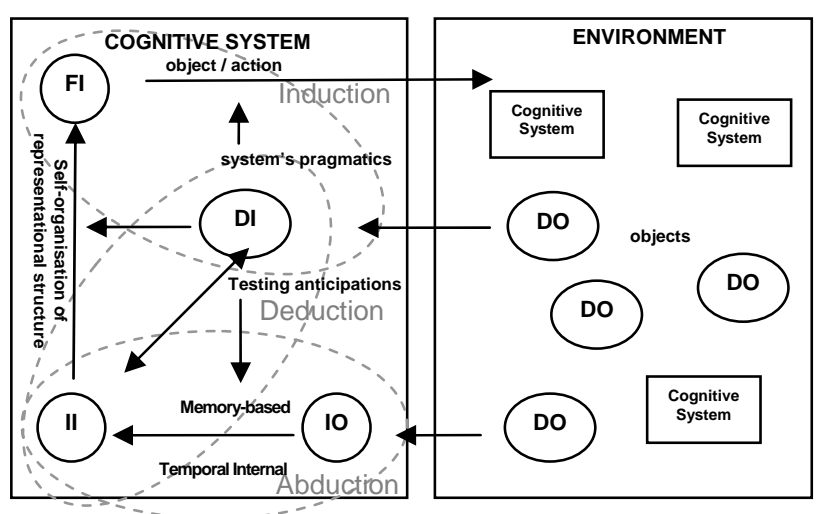

Figure 1. An agent engaging in self-organising semiotic processes with the environment.
Moreover, since each agent interacts in a different manner with the environment, similar actions will correspond to different representational structures.

A perception mechanism, which is constantly being informed by the environment, about the entities that are in its field of view, and performs the required data transformations to create the IOs as components that will drive the semiotic process. These IOs are stored in the short term memory, which agents are constantly examining and comparing to their representational structure to try and detect any surprising phenomena, i.e. objects that they cannot categorize. In the implemented example, the semiotic components describe entities, spatial relations, situations and actions. Surprising phenomena could be the detection of a new entity or an unanticipated change in successive situations. In the first case, the new IO is stored in the representational structure, while in the second one, the process of semiosis is initiated in order to explain the action that resulted in the unexpected change, causing the creation of a new category of actions or the restructuring of an existing one as it is shown in Fig. 2.

Let us consider the case where two successive situations are observed by the agent and an action component is being created. This component describes the first situation as possible preconditions of the action and the differences between the two as the anticipated distinctions (Fig. 3). This component should be compared to all known actions in the representational structure to check whether it is an instance of a known category. The process of testing for similarities between an action that has happened and a generic category of actions is as follows: First, if the number of entities that take part in the action effects is different, the agent concludes that there are no similarities. Otherwise, all possible permutations of the entities of the current action are replacing the respective entities of the DI. Differences are tracked, and the arrangement with the least number of differences is determining the similarity score, i.e. a number stating how similar the observed action is to that category in the representational structure. In this case, one may have to find differences between values and sets, so there is a difference if a quality value of the observed action does not belong to the set of values of the generic one.

Failure to categorize an observed situation drives the process of semiosis. A completed semiosis consists of the three inferential procedures: abduction, deduction and induction, which drive the agent's logical argumentation.

\section{Abductive Phase}

The first part of abduction consists of the observation and description of the nature of a surprising phenomenon on the basis of the anticipations of the agent. 


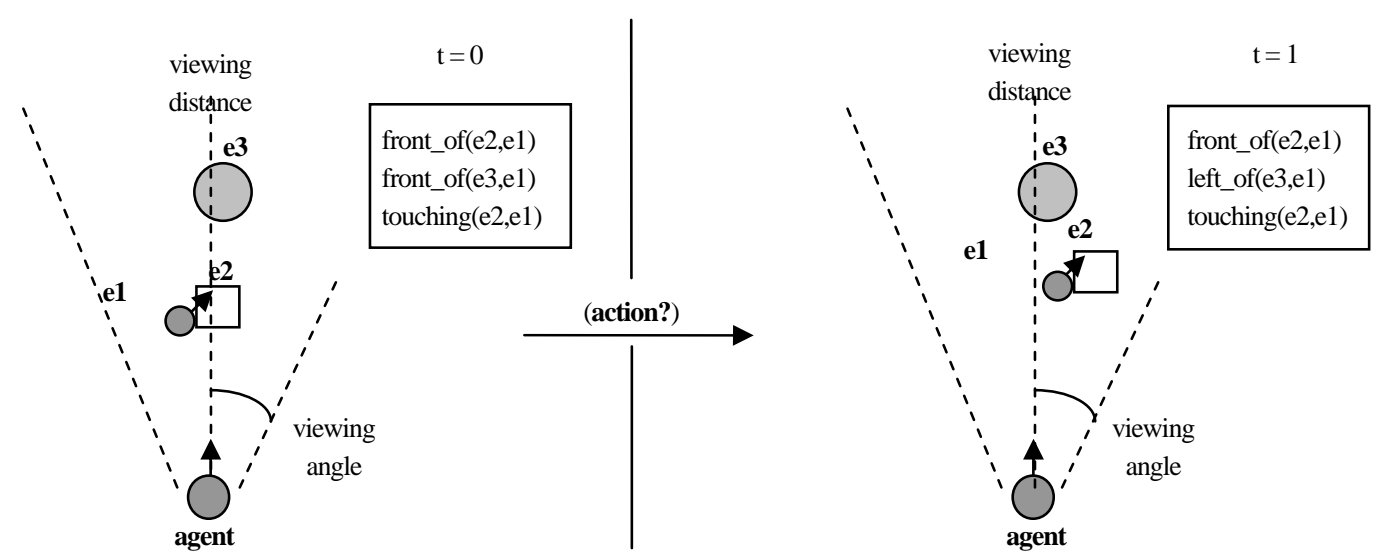

Figure 2. Indication of a possible action. The agent has observed differences in qualities and relations in two successive situations.

Hence, the interaction initiates from the dynamic object (DO), the environmental element of interaction, which needs mediation in order to be determined in a representation of the cognitive system. A direct relation between the DO and its interpretant is impossible, but, using a means of measurement, the nature of the DO can be indicated by a primitive internal representation, the immediate object (IO). At this moment of the interaction, the sign, specifically the representamen, indicates the direction of the reality to which it refers. A representamen contains several IOs which in turn refer to several DOs. Which IO will eventually be actualized depends on the cognitive system's anticipations. This is where semantics play their role. They make use of the given information in order to infer its meaning, but only in the sense of the ground of the representation. As the form of the ground is a function of the qualities of the representamen, the latter constraints the former, which sets the borders for the structure of the impending representation.

In the example, the abductive phase initiates whenever the categorization of an immediate object fails. The decision made after that is determined by the highest similarity score. If it reaches below a certain threshold, the IO is treated as belonging to a new category and is stored in the representational structure. In the opposite case, the agent tries to restructure the existing category so as to explain the observed object. A new semiotic component is created that contains all differences between the IO and the category with the most similarity.

In the second part of the abduction, an analogy between the surprising phenomenon and the agent's anticipations is attempted, in order to indicate a possible direction of a hypothesis explaining the surprising phenomenon. Hence, further to IO's formation, the result is tested against the system's anticipations, where an interpretation of the qualities of the representamen in a way narrow the IO's selection and give a certain directionality to the system.

The restructuring is being driven by a possible hypothesis that generalizes or specializes the object description (DI). E.g. if there are differences between an observed action $a$ and a known category $A$, there are two possible hypotheses that could explain such a surprising phenomenon: either $a$ is a new case of $A$, so the category has to be generalized to include the differences, or $a$ is an exception of $A$, so the category has to be specialized to exclude one or more qualities of $a$. The differences between $a$ and $A$ can be the following:

- differences in preconditions: quality values that do not match, different relations, or different number of entities.

- differences in anticipated distinctions: quality differences that do not match, different relations in the added relations list, or different relations in the removed relations list.

At the final part of the abduction a formulation of a possible explanation for the surprising phenomenon takes place. At this point the immediate interpretant (II) has been formed. It should be noted that for II's formation system's semantics must be tested against the pragmatics for the ground to be enriched with -not all possible- but only system's relevant and useful (at the specific moment) predicates. In the whole abductive phase indexical and iconic sign types would be formed. The indexical representations should be considered as digital representations, where their information capacity coincides with their representational content. 


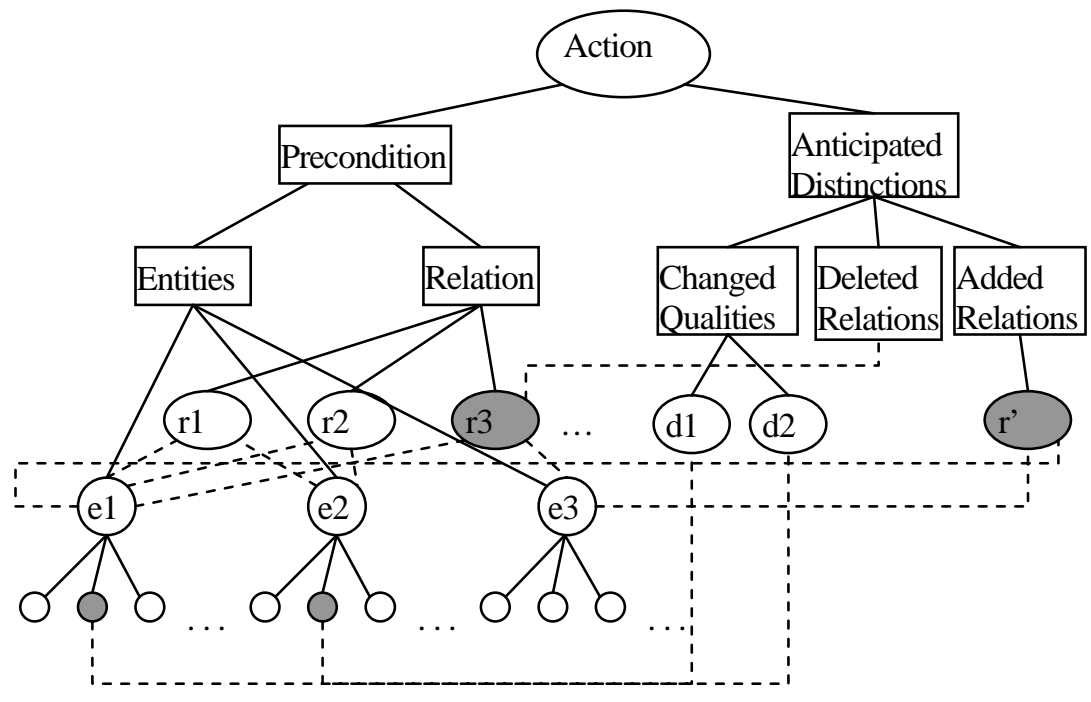

Figure 3. An action as a semiotic component.

These representations cannot provide the agent with new knowledge for its objects are related to existence. On the other hand, iconic sign types should be considered as analog representations able to produce new knowledge since their information capacity is more that their representational content.

In the presented example, using the semiotic component that marks the differences, one is randomly selected and, based on the hypothesis, a possible generalization or specialization is selected. In the end of the abductive phase, $A^{\prime}$ is created as a copy of $A$ to describe the revised category if the hypothesis were true.

\section{Deductive Phase}

In the deductive phase the consequences of the hypothesis formulated in the abductive phase are examined. In the first part of deduction, a possible direction of the consequences of the hypothesis is indicated based on the agent's anticipations. In the second part, the formulation of the consequences of the hypothesis takes place. Hence, although the representational content of the II provided the tools for the object's discrimination, there will be some tests needed in order this core meaning to be temporarily stabilized into a dynamic interpretant (DI). This process is the most complicated one as the self-organised system will try to incorporate the new representational structure (II) in its functional organisation. To do this, the system must refer to itself in order for unsuccessful structure modifications to be obliterated. Certain representations may not be fulfilled by their objects, and this amounts to improper expectations. These could lead to omissions and commissions. The former implies an unanticipated behaviour of the object, while the latter implies that the agent will not be able to manipulate the object in the anticipated way. This is the reason for incorporating the pragmatic aspect of the representation.

In the example, during the deductive phase the effects of the hypothesis are applied to $A$ '. The generalization mechanisms are: to expand the set of quality values so as to include the different values of $a$, to remove a relation that does not exist in $a$, or to remove an entity that does not take part in $a$. On the other hand, a specialization is to pickup a random quality of $a$, whose value belongs to the set of values of the respective quality of $A$, and to reconstruct the set so as to exclude that value. Table 2 summarizes the possible actions that can take place to restructure an action category in the agent's representational structure, based on the type of difference and the hypothesis.

Both $A$ and $A^{\prime}$ are kept in memory and linked to each other as $A^{\prime}$ is a descendant action of $A$. So, based on the results of the abductive phase, a surprising phenomenon will either create a new tree as a single node, or expand an existing tree by adding a descendant node to the most similar of its nodes.

\section{E. Inductive Phase}

The, in a way, objective meaning, which results from the semantic and pragmatic processes, should be open to revision, which takes place in the inductive phase. The consequences of the hypothesis formulated in the deductive phase are observed in the context of the surprising phenomenon. In case of acceptance, the hypothesis can be used to account for similar surprising phenomena in the future. Then, a new belief would be fixed and if such a hypothesis continues to persist through the agent's interaction with the environment, it will grow to a habit. The final interpretant (FI) is an ideal case where a representational structure coincides with the intentionality of the respective object (see Fig. 1).

The inductive mechanism of the agent in the example operates as follows: If the perceived context meets the preconditions of an action, and that action involves at least one entity of type 'agent', the agent's behavior tries to imitate the action. This imitation takes place by trying to reproduce the action's effects. For example, if there is an anticipated change in the agent's position, the agent actually changes its position in order to meet the changes in the action effects. It uses a simple mechanism to get feedback from the actions it observes: each action in memory is assigned a score, and, whenever it is observed 
in the environment or it is the most similar to an action observed in the environment, its score is increased and the score of all other nodes in the same category tree is decreased. The nodes whose score is below a certain threshold are deleted from memory. With this process the agent manages to test its hypotheses by trying them, and to reinforce the correct ones, leading to the restructuring of its representational structure.

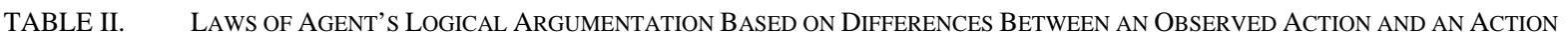
CATEGORY

\begin{tabular}{|c|c|c|c|}
\hline Difference & Hypothesis & Direction & Actions \\
\hline \multirow{2}{*}{$\begin{array}{c}\text { Different } \\
\text { Preconditions }\end{array}$} & $a \in A$ & Generalize preconditions & Quality difference $\rightarrow$ expand set \\
\cline { 3 - 4 } & & & Relation difference $\rightarrow$ remove relation \\
\cline { 3 - 4 } & $a \notin A$ & Specialize effects & Entity difference $\rightarrow$ remove entity \\
\hline \multirow{2}{*}{$\begin{array}{c}\text { Different } \\
\text { Effects }\end{array}$} & $a \in A$ & Generalize effects & Quality difference $\rightarrow$ expand set \\
\cline { 3 - 4 } & $a \notin A$ & Specialize preconditions & Relation difference $\rightarrow$ remove relation \\
\cline { 4 - 4 } & \multirow{2}{*}{$a$} & & Exclude quality value from set \\
\hline
\end{tabular}

\section{CONCLUSIONS AND FUTURE WORK}

We have presented an example as an application of the proposed framework, where agents evolve representational structures regarding new actions by observing the environment and trying to interact with it. Each agent in the environment creates its own categories of actions based on its experiences. The emergent representational structures could be reused and adapted to other environments with similar physics. Furthermore, the frame-like structure of the semiotic elements allows the representational structure of an agent to be stored and corrected offline by a designer, e.g. to remove possible false hypotheses about actions.

The structure of the Peircean semiotic processes overcome the symbol grounding problem as they are already grounded by their nature. The fact that a representamen mediates between the DO and its interpretant provides a Peircean semiotic process with an embodied structure, since now agent's anticipations are grounded in agent-environment interaction.

The richness of the proposed framework points to a variety of directions that could be pursued in the future. The authors plan to extend the analysis and implementation in more complex environments, where the representation of actions could involve more than two successive timeframes. This would allow agents to anticipate long-term actions, as well as their performance, i.e. the series of micro actions needed to accomplish them, thus embedding the agent seriously into time and enriching its degree of representational autonomy and consequently its autonomy and adaptability. More important future questions that this framework raises are related to efficient inference and planning of the agent based on the emergent representational structures, as well as to the organisation of higher level action and goal structures in a social context.

\section{REFERENCES}

[1] R. Aylett and M. Cavazza, "Intelligent Virtual Environments: a State-of-the-Art Report,” In: Eurographics, STAR Reports volume, 2001.

[2] J. Collier, "Autonomy in Anticipatory Systems: Significance for Functionality, Intentionality and Meaning," in the 2nd Int. Conf. on Computing Anticipatory Systems, D. M.Dubois, Ed. Springer-Verlag, New York, 1999

[3] W. D. Christensen and C. A. Hooker, “Organised interactive construction: The nature of autonomy and the emergence of intelligence", in The Contribution of Artificial Life and the Sciences of Complexity to the Understanding of Autonomous Systems, Communication and Cognition, A. Moreno, et al. Eds, Special Edition, 2000.

[4] W. D. Christensen and C. A. Hooker, "Representation and the Meaning of Life," in Representation in Mind: New Approaches to Mental Representation, H. Clapin, P. Staines, and P. Slezak, Eds, Oxford: Elsevier, 2004.

[5] A. Guye-Vuillieme and D. Thalmann, "A High Level Architecture For Believable Social Agents,” VR Journal, Springer, vol.5, pp. 95-106, 2001.

[6] J. A. R. Torres, L. P. Nedel, and R. H. Bordini, "Using the BDI architecture to produce autonomous characters in virtual worlds,” LNAI (2792), Springer-Verlag, 2003, pp. 197-201.

[7] S. Harnad, “The Symbol Grounding Problem,” Physica, D 42, 1990, pp. 335-346.

[8] T. Ziemke, "Rethinking Grounding," in Understanding Representation in the Cognitive Sciences Riegler, Peschl and von Stein, Eds, Plenum Press, New York, 1999.

[9] R. A. Brooks, "Intelligence Without Representation," Artificial Intelligence, 47, pp. 139-159, 1991.

[10] M. Anderson, "Embodied Cognition: A field guide", Artificial Intelligence, 149 (1), pp.91-130, 2003. 
[11] R. A. Brooks, "From Earwigs to Humans“, Robotics and Autonomous Systems, 20, pp. 291-304, 1997.

[12] R. Pfeifer, C. Scheier, Understanding Intelligence, MIT Press, Cambridge, M.A. 1999.

[13] J. Funge, X. Tu, D. Terzopoulos, “Cognitive Modeling: Knowledge, Reasoning and Planning for Intelligent Characters," SIGGRAPH 1999, pp. 29-38.

[14] B. Blumberg, M. Downie, Y. Ivanov, M. Berlin, M.P. Johnson, and B. Tomlinson. "Integrated learning for interactive synthetic characters," in Proceedings of the ACM SIGGRAPH, 2002.

[15] H. R. Maturana and F. J. Varela, Autopoiesis and Cognition: The Realization of the Living, Boston: Reidel, 1980.

[16] P. Cariani, "Symbols and Dynamics in the Brain," BioSystems. 60, pp. 59-83, 2001.

[17] S. Brier, "The Construction of Information and Communication: A Cybersemiotic Re-entry into Heinz von Foerster's Metaphysical Construction of Second-Order Cybernetics," Semiotica, 154-1/4, pp. 355-399, 2005.

[18] C. S. Peirce, The Essential Peirce. Selected Philosophical Writings, Vol. 1 (1867-1893), N. Houser and Ch. Kloesel, Eds. Vol. 2 (1893-1913) ed. by the Peirce Edition Project, Bloomington and Indianapolis: Indiana University Press, 1992 and 1998.

[19] A. Arnellos, T. Spyrou, and J. Darzentas, "Towards a Framework that Models the Emergence of Meaning Structures in Purposeful Communication Environments", The $47^{\text {th }}$ Annual Conf. of the Int. Society for the Systems Sciences (ISSS) 3 (103), 2003.

[20] P. Vogt, "The emergence of compositional structures in perceptually grounded language games”, Artificial Intelligence 167 (1-2), pp. 206-242, 2005.

[21] D. Roy, "Semiotic Schemas: A Framework for Grounding Language in the Action and Perception," Artificial Intelligence, 167 (1-2), pp 170-205, 2005.

[22] E. Thompson, "Symbol Grounding, "A Bridge from Artificial Life to Artificial Intelligence," Brain and Cognition, 34, pp. 48-71, 1997.

[23] A Arnellos, T. Spyrou, and J. Darzentas, "Dynamic Interactions in Artificial Environments: Causal and NonCausal Aspects for the Emergence of Meaning,” accepted for publication in the Journal of Systemics, Cybernetics and Informatics, International Institute of Informatics and Cybernetics, 2006. 